\title{
As redes sociais de apoio no cuidado às pessoas com estomias: Revisão \\ Bibliográfica
}

\author{
Sandra Ost Rodrigues* Maria de Lourdes Denardin Budó** Bruna \\ Sodré Simon ${ }^{\star \star *}$ Melissa Gewehr*** Dalva Cézar da Silva***
}

\begin{abstract}
Resumo: Objetivo - conhecer e analisar a produção científica sobre as redes sociais de apoio no cuidado às pessoas com estomia. Método: Revisão bibliográfica, de abordagem qualitativa, com busca nas bases de dados Literatura Latino-Americana e do Caribe em Ciências da Saúde e Medical Literature Analysis and Retrieval System Online, usando como palavras-chave: "estomia", "apoio" e "social", sem recorte temporal. Resultados - A partir da análise de 11 artigos, emergiram as categorias: Apoio familiar; Apoio da associação e grupos de pessoas com estomias; e Apoio realizado pelo enfermeiro e outros profissionais da saúde. Os estudos qualitativos foram os mais frequentes e a produção científica de enfermagem numericamente pequena. Conclusão - Concluiu-se que 0 conhecimento sobre a rede social de apoio é importante para o cuidado às pessoas com estomias.
\end{abstract}

Descritores: Apoio social; Estomia; Enfermagem; Cuidados de Enfermagem.

\section{Support social nets of ostomy persons care: Bibliographic Revision}

Abstract: Objective - knowing and analyzing the scientific production about support social nets of ostomy person's care. Method: Bibliographic revision, with qualitative approach, searching in databases Latin-American and Caribbean Health Sciences Literature and Medical Literature Analysis and Retrieval System Online, using as key-words: "ostomy", "support" and "social", without a temporal cut. Results From the analysis of 11 articles, have emerged the following categories: Family support; Association and Groups of persons with ostomies Support; and Nurse and other health professionals Support. The qualitative studies were the most frequent and nursing scientific production numerically small. Conclusion - It was concluded that the knowledge about support social net is important for persons with ostomies care.

Descriptors: Social support; Ostomy; Nursing; Nursing Care.

*Mestre em Enfermagem pela Universidade Federal de Santa Maria (UFSM), Santa Maria, RS, Brasil ${ }^{*}$ Doutora em Enfermagem pela Universidade Federal de Santa Catarina (UFSC), Florianópolis, SC, Brasil

***Mestranda em Enfermagem na Universidade Federal de Santa Maria (UFSM), Santa Maria, RS, Brasil 


\section{Introdução}

As pessoas que sofrem algum agravo à saúde que necessitam submeter-se a um procedimento cirúrgico para eliminar o conteúdo fecal através da parede abdominal, enfrentam dificuldades psicológicas, sentimentais, principalmente pela mudança de seus hábitos de vida ${ }^{1}$. Dentre as transformações decorrentes dessa condição há a incorporação de novos hábitos e adaptação dos papéis sociais desenvolvidos por eles².

O vocábulo "estoma" tem origem grega e é uma abertura cirúrgica no abdômen, onde os dejetos são expelidos quando a função normal do intestino é interrompida. As estomias recebem nomes diferenciados: no intestino grosso - colostomia; no delgado - ileostomia ${ }^{3}$. No decorrer deste trabalho adotou-se o termo "estomia" por se tratar do termo adotado pela Associação Brasileira de Estomaterapia.

Além dos problemas comumente enfrentados pelas pessoas submetidas a uma cirurgia, os estomizados enfrentam a exposição a uma série de constrangimentos sociais, acarretados pelo uso da bolsa coletora, com possibilidades de eliminação de gazes ou falha na própria bolsa. Resultando, assim em um isolamento social grave ${ }^{1}$. Exigindo, consequentemente um cuidado holístico, que envolva principalmente sua rede de relações. Destaca-se que este cuidado pode ser ofertado pela família, amigos, membros de congregações religiosas e grupos de apoio ${ }^{4}$.

Uma rede social consiste na estrutura a partir da qual advém o apoio social, ou seja, é o conjunto dos vínculos relacionados ao indivíduo, quer por laços de parentesco, amizade ou conhecidos; ou ainda um quadro de relações de um indivíduo em particular ou; um quadro de ligações entre um grupo de pessoas ${ }^{5}$.

Por essa razão, é um desafio aos profissionais de saúde, entre eles o enfermeiro, trabalhar com as singularidades e complexidades dos contextos de vida das pessoas com estomia. Isto se torna relevante, pois a confecção de uma estomia é considerada um acontecimento traumático, que causa alterações profundas no modo de vida ${ }^{6}$, tanto no âmbito biológico, como psicológico, social, cultural e espiritual ${ }^{7}$.

Além do atendimento profissional, essencial para reabilitação, destaca-se que 0 apoio encontrado na família, amigos e vizinhos contribui na adaptação da pessoa com estomia. Esses, associados com a religiosidade e apoio dos grupos operativos tornam-se importantes para o processo de conviver nesse novo cenário de vida ${ }^{8}$. Evidencia-se assim, a necessidade das inter-relações sociais ${ }^{6-8}$.

Logo, acredita-se, que o conhecimento sobre a rede social de apoio no cuidado à pessoa com estomia auxilia na melhora da qualidade de vida e permite que essas consigam enfrentar esta situação. Para tanto, faz-se necessário que profissionais da saúde conheçam e valorizem essas redes, a fim de visualizar a pessoa com estomia e seu contexto social.

Frente a isso, questiona-se o que se tem produzido acerca das redes sociais de apoio no cuidado às pessoas com estomia? Portanto, objetiva-se conhecer e analisar a produção científica sobre as redes sociais de apoio no cuidado às pessoas com estomia.

Saúde (Santa Maria), v.39, n.1, p. 33-42, 


\section{Método}

Pesquisa bibliográfica realizada em janeiro de 2012, nas bases de dados Literatura da América Latina e Caribe em Ciências da Saúde (LILACS) e Medical Literature Analysis and Retrieval Sistem On-line (MEDLINE).

Para estratégia de busca, utilizou-se a associação das palavras-chave: "estomia", "apoio" e "social", uma vez que o emprego de descritores dessa temática limitou os achados. Utilizou-se o operador booleano "and" sem critério de recorte temporal. Os critérios de inclusão foram ser artigo, disponível na íntegra on line, em inglês, português ou espanhol. Optou-se como exclusão os artigos sem resumo ou incompletos.

Conforme a estratégia de busca foram encontradas 47 publicações no total, sendo destas, 10 na LILACS e 37 na MEDLINE. Diante das repetições nas bases de dados, os critérios de inclusão e exclusão, o corpus desta pesquisa ficou constituído por 11 artigos que foram analisados na íntegra.

Os artigos selecionados foram trabalhados com base nas três etapas da análise de conteúdo temático: pré-análise, exploração do material, tratamento e interpretação dos resultados ${ }^{10}$.

Utilizou-se um quadro sinóptico para identificar os artigos quanto ao ano, país, área do conhecimento e tipo de pesquisa. Após análise do corpus da pesquisa emergiram como categorias: Apoio familiar; Apoio da associação e grupos de pessoas com estomias e Apoio realizado pelo enfermeiro e outros profissionais da saúde.

\section{Resultados}

As características dos 11 artigos analisados apresenta-se no quadro I, o qual contém a descrição quanto ao país de publicação, ano, revista, tipo de artigo e base de dados.

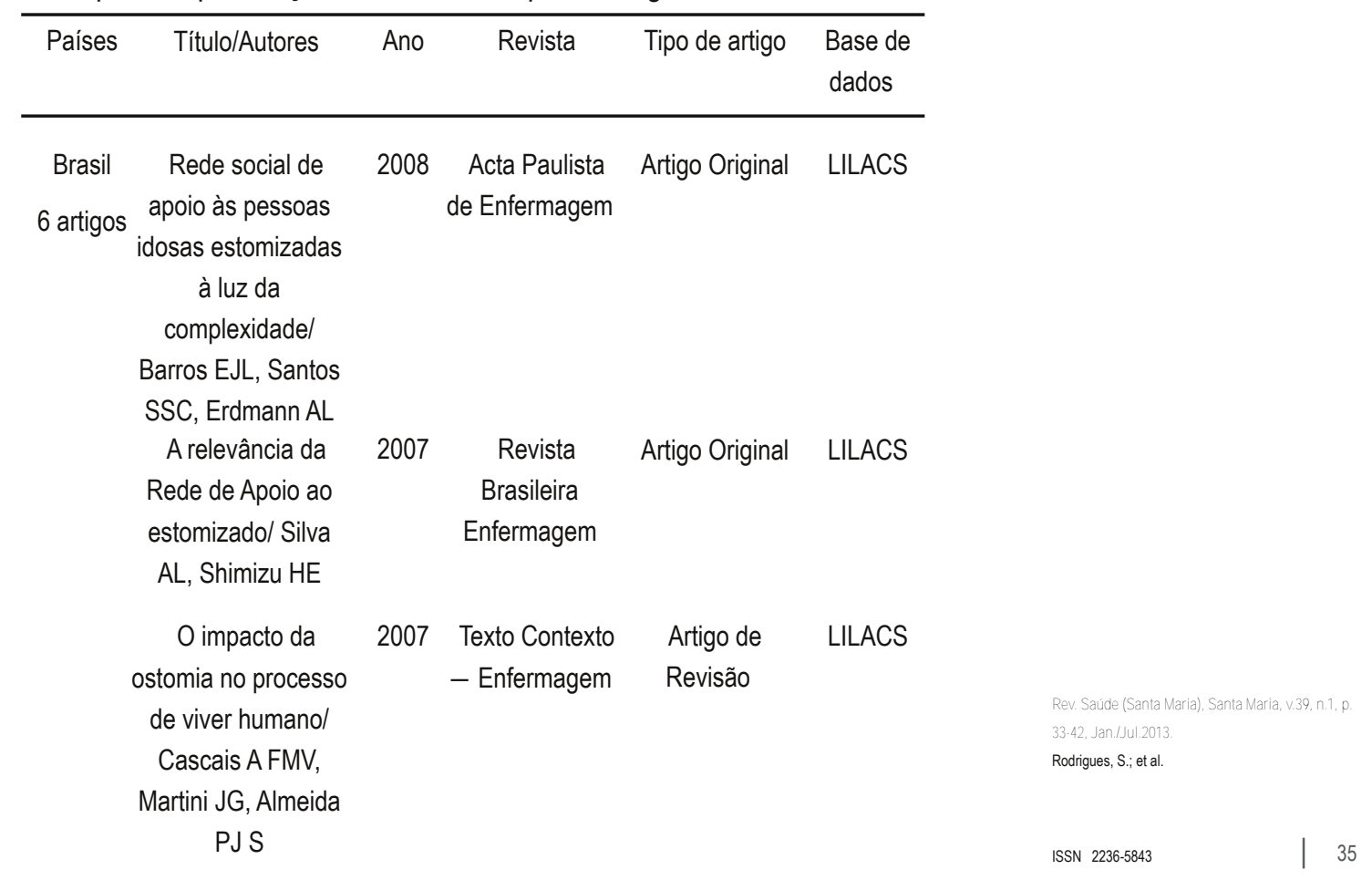




\begin{tabular}{|c|c|c|c|c|c|}
\hline \multirow[t]{3}{*}{$\begin{array}{l}\text { Brasil } \\
6 \\
\text { artigos }\end{array}$} & $\begin{array}{c}\text { O significado da } \\
\text { mudança no modo } \\
\text { de vida da pessoa } \\
\text { com estomia } \\
\text { intestinal definitiva/ } \\
\text { Silva A L, Shimizu H } \\
\text { E }\end{array}$ & 2008 & $\begin{array}{l}\text { Revista Latino- } \\
\text { Americana de } \\
\text { Enfermagem }\end{array}$ & Artigo Original & LILACS \\
\hline & $\begin{array}{c}\text { A trajetória do } \\
\text { grupo de apoio à } \\
\text { pessoa ostomizada: } \\
\text { projetando ações em } \\
\text { saúde e } \\
\text { compartilhando } \\
\text { vivências e saberes/ } \\
\text { Martins ML, Silva } \\
\text { RDM }\end{array}$ & 2005 & $\begin{array}{l}\text { Texto contexto } \\
\text { - Enfermagem }\end{array}$ & $\begin{array}{l}\text { Relato de } \\
\text { experiência }\end{array}$ & LILACS \\
\hline & $\begin{array}{c}\text { Parceria - uma } \\
\text { estratégia para } \\
\text { promoção da saúde/ } \\
\text { Trentini M, Silva D, } \\
\text { Pacheco M, Martins } \\
\text { M. }\end{array}$ & 1996 & Cogitare & $\begin{array}{l}\text { Relato de } \\
\text { experiência }\end{array}$ & LILACS \\
\hline \multirow[t]{3}{*}{$\begin{array}{l}\text { England } \\
\qquad 3 \\
\text { artigos }\end{array}$} & $\begin{array}{l}\text { Managing quality of } \\
\text { life in the older } \\
\text { person with a stoma/ } \\
\text { Slater RC }\end{array}$ & 2010 & $\begin{array}{l}\mathrm{Br} \mathrm{J} \\
\text { Community Nurs }\end{array}$ & Artigo original & MEDLINE \\
\hline & $\begin{array}{l}\text { Continuity of care } \\
\text { for the stoma } \\
\text { patient: } \\
\text { psychological } \\
\text { considerations/ } \\
\text { Borwell B }\end{array}$ & 2009 & $\begin{array}{c}\mathrm{Br} \mathrm{J} \\
\text { Community Nurs }\end{array}$ & $\begin{array}{l}\text { Artigo de } \\
\text { revisão }\end{array}$ & MEDLINE \\
\hline & $\begin{array}{l}\text { Stoma care } \\
\text { nursing: what the } \\
\text { community nurse } \\
\text { needs to know/ } \\
\text { Williams J }\end{array}$ & 2007 & $\begin{array}{l}\mathrm{Br} \mathrm{J} \\
\text { Community Nurs }\end{array}$ & $\begin{array}{l}\text { Artigo de } \\
\text { revisão }\end{array}$ & MEDLINE \\
\hline
\end{tabular}

Saúde (Santa Maria), v.39, n.1, p. 33-42, 


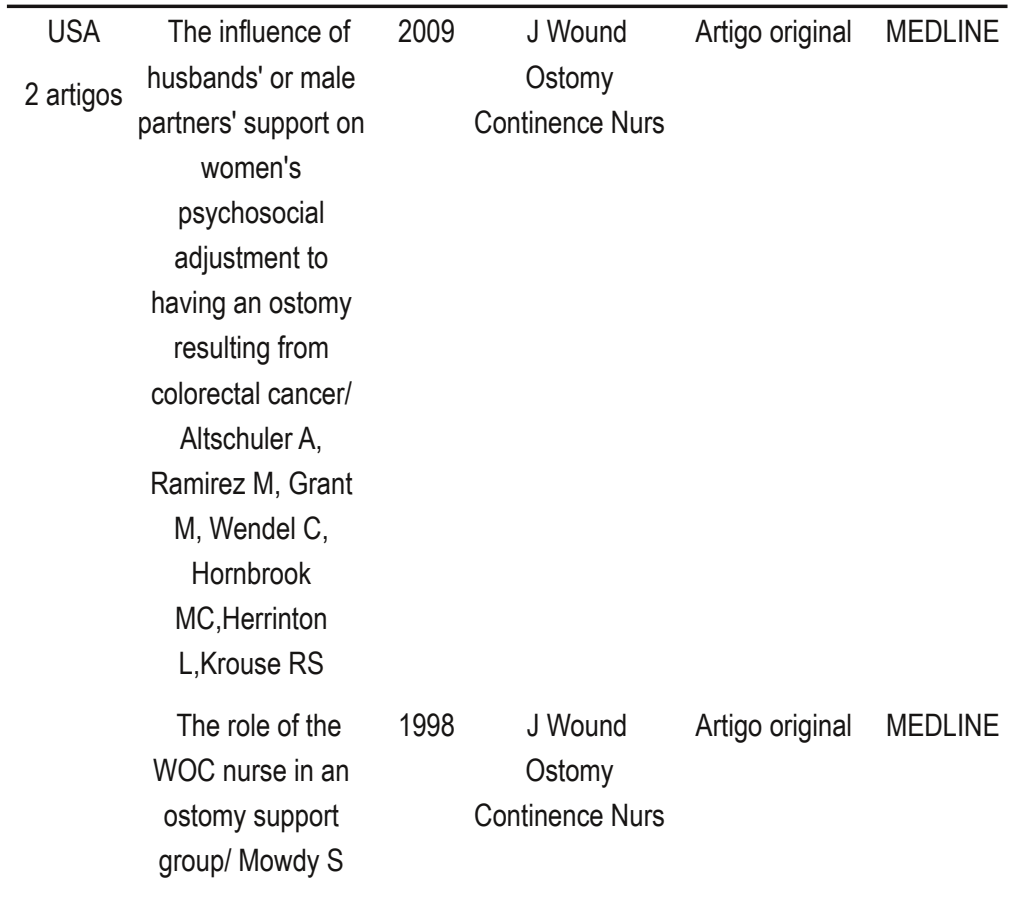

Quadro I: Apresentação das publicações sobre redes sociais de apoio no cuidado às pessoas com estomia, 2012.

A maioria dos artigos é de origem brasileira, sendo todos os artigos nacionais de autoria de enfermeiras. Como local dessas pesquisas encontrou-se que em dois artigos o cenário foi - Serviço Ambulatorial de Enfermagem em Estomaterapia do Hospital Universitário de Brasília e um o domicílio da paciente com estomia, na cidade de Fortaleza, Ceará. Os dois relatos de experiência e uma revisão têm como autoras enfermeiras do estado de Santa Catarina.

A abordagem sobre as redes sociais de apoio à pessoa com estomia ocorre de maneira diferenciada nas produções. Por meio da análise dos artigos emergiram categorias que se referem às principais questões abordadas sobre as redes sociais de apoio no cuidado à pessoa com estomia, sendo o Apoio familiar; Apoio da associação e grupos de pessoas com estomias; e Apoio realizado pelo enfermeiro e outros profissionais da saúde.

\section{Apoio familiar}

Nesta categoria, o apoio familiar recebe destaque, uma vez que a família influencia na recuperação e na aceitação dessa nova situação vivenciada pela pessoa com estomia ${ }^{6,11-14}$. Alguns autores referem a influência do apoio dos homens no ajustamento psicossocial das mulheres com estomia resultante de câncer colorretal. Em uma pesquisa realizada com 22 mulheres casadas ou com parceiro, 17 consideram 0 apoio dos maridos sendo essencial para a sua adaptação psicossocial, três relatam ser negativo não ter 0 apoio dos maridos, $\mathrm{e}$ duas apontam o suporte como indiferente. Sugere-se que ter ou não os maridos ou parceiros como suporte pode influenciar consideravelmente no ajustamento psicossocial das mulheres com estomias ${ }^{11}$.

Um estudo refere que a família oferece apoio, carinho e atenção em todas as fases da doença, tendo o compromisso de acalentar, confortar, ajudar, todos envolvidos pelo vínculo da afetividade ${ }^{14}$. Nesse sentido, a família pode ser fundamental e determinante na aceitação Rev. Saúde (Santa Maria), Santa Maria, v.39, n.1, p. 33-42, Jan./Jul.2013. da estomia e consequentemente para o seu processo de reabilitação e adaptação ${ }^{6}$. 
Uma pesquisa do tipo estudo de caso, que enfoca a aplicação da Teoria de Orem, menciona a evidência teórica, prática e investigacional do significado da família, tanto para 0 bem-estar e a saúde dos seus membros, como para a influência sobre a doença, fazendo com que as enfermeiras sempre considerem o cuidado centrado na família como parte integrante do cuidado de enfermagem ${ }^{12}$.

Autores evidenciam ainda que a família desenvolva um papel fundamental no processo de recuperação do paciente, bem como na aceitação de sua condição, quando esta demonstra sentimento de fé e esperança, criando mecanismos de união e força entre seus integrantes. Destaca-se a família como elemento importante em todo o processo da vida, antes e depois da estomia, por facilitar a integração da experiência da doença na biografia dos portadores ${ }^{13}$.

\section{Apoio da associação e grupos de pessoas com estomias}

$O$ apoio ofertado pelos grupos e associações foi destacado em diversos estudos ${ }^{13-18}$.

Esses grupos emergem como um ambiente de aprendizagem e como um espaço de troca de experiências entre profissionais e as próprias pessoas que possuem estomias ${ }^{15}$. A convivência nesses locais pode favorecer a aceitação da pessoa à nova situação, bem como despertar nos participantes 0 interesse pela luta por seus direitos ${ }^{18}$.

Nos artigos analisados, destacou-se o estado de Santa Catarina pela sua história na organização desses grupos ${ }^{18}$. Um trabalho descreve que essa convivência em grupos ou associações como uma rede de apoio tem como objetivo ações de saúde fundamentadas na parceria, que se expressam por meio da troca de vivências e saberes entre profissionais, usuários e familiares ${ }^{16-18}$. Outro trabalho enfatiza que a proposição adotada pelo grupo destaca a pessoa na perspectiva de sua multidimensionalidade, unicidade, integralidade, engajada no mundo como agente de sua história, assim diferenciando do paradigma biomédico ${ }^{16}$.

Ao participarem dos grupos, essas compartilham informações sobre o cuidado por meio de conversas, bem como percebem que as relações sociais podem ser construídas ${ }^{15}$. Assim, 0 apoio emocional recebido no grupo de apoio auxilia a superar o sentimento de isolamento ${ }^{17}$.

Dois artigos estudados referem sobre os grupos na Associação de Estomizados, destacando como um lugar importante na vida das pessoas com estomia, pois representa um espaço onde eles conversam sobre diversos assuntos, como a vida familiar e social, além de terem acesso a diversos recursos materiais e informações sobre 0 autocuidado ${ }^{13-14}$.

Apoio realizado pelo enfermeiro e profissionais da saúde em geral

Outro apoio identificado nesta pesquisa foi 0 ofertado pelos enfermeiros e outros profissionais de saúde $\mathrm{e}^{13-15-17-19-20}$.

A estrutura de atendimento profissional é essencial para a reabilitação e adaptação da pessoa com estomia ${ }^{13}$, principalmente representado pelo apoio do enfermeiro.

A aceitação da imagem corporal e alterações no estilo de vida da pessoa com estomia pode ser influenciado pelo apoio que recebe do enfermeiro e de outros profissionais de saúde envolvidos no período transoperatório de confecção da estomia. Sabe-se que o preparo no pré-operatório, por meio da escuta das necessidades individuais favorece a percepção de aceitar a estomia ${ }^{15}$. Dessa forma, o enfermeiro precisa conhecer o que a pessoa cuidada percebe como qualidade de vida, para assim, alterar os fatores que podem inibir a capacidade de cuidar-se cuidar e aceitar a estomia ${ }^{15}$. 
A pessoa com estomia e sua família podem apresentar sentimentos contraditórios a respeito de como eles vão lidar com a existência da estomia ${ }^{19}$. A comunicação eficaz e a colaboração dos profissionais de saúde são fundamentais para a adaptação e reabilitação, na transição do hospital para casa após o procedimento cirúrgico. A cirurgia de confecção do estoma pode afetar negativamente a autoimagem dos pacientes, dessa maneira o enfermeiro pode colaborar para o enfrentamento destas mudanças ao voltar para o domicílio ${ }^{20}$.

Na maioria dos casos, após a alta do hospital, o enfermeiro estomaterapeuta pode realizar visitas domiciliárias como meio de manter a continuidade dos cuidados ${ }^{20}$. Além disso, pode atuar no grupo de apoio aos estomizados, ofertando orientações e estimulando para repercussão no seu contexto de vida ${ }^{17}$.

\section{Discussões}

Frente aos achados, as relações familiares têm papel preponderante no processo de reabilitação da pessoa com estomia, podendo minimizar ou maximizar as consequências advindas da estomia ${ }^{13}$. Sendo assim, o profissional deve motivar o familiar a ter uma atitude positiva frente à pessoa com estomia.

0 apoio emocional fornecido pela família manifesta-se por meio de diálogos, da presença, do estar disponível, transmitindo conforto e segurança, ressaltando os aspectos positivos da estomia e auxiliando na aceitação e na diminuição dos medos e angústias ${ }^{21}$. A família é caracterizada pelas pessoas com estomias como sendo uma fonte de apoio, ajuda física e emocional, durante a trajetória da confecção da estomia e principalmente no processo de reabilitação e adaptação desta nova fase de vida.

O cuidado de enfermagem engloba a percepção dos diferentes cenários que integram a vida da pessoa com estomia, conhecendo sua rede social de apoio, seu cuidador, sua família, pois esses podem favorecer o preparo da pessoa para as ações de cuidado ${ }^{12}$. A compreensão do benefício que o núcleo familiar proporciona à pessoa com estomia, leva o profissional a valorizar o engajamento da família no processo de cuidado, bem como no cuidar de si. Nesta perspectiva, se ela também receber cuidado e for potencializada para o desenvolvimento deste, terá condições de cuidar de seu familiar ${ }^{21}$.

O profissional deve planejar suas ações condizentes com a realidade de cada pessoa com estomia e sua família, tornando-se, dessa forma um ligante entre os familiares e essa pessoa, no estabelecimento do vínculo e confiabilidade ${ }^{4}$. Reitera-se que há uma necessidade de envolvimento do profissional na ajuda terapêutica, mediada pela comunicação. Esta se caracteriza como um dos papéis da enfermagem relevantes durante as orientações do transoperatório e da continuidade da assistência ${ }^{4}$.

As orientações ofertadas buscam esclarecer as dúvidas inerentes à função da estomia, seus cuidados e retomada das atividades diárias. A realização dessas orientações, por meio de uma comunicação clara e objetiva, resulta em cuidado a pessoa com estomia e pode favorecer na aceitação do novo modo de viver ${ }^{19}$. A troca de saberes e práticas permitem que, em uma relação de parceria, se estabeleçam estratégias que contribuam, gradativamente, para a melhoria na qualidade de vida tanto da pessoa com a estomia, quanto na de seus familiares.

Rev. Saúde (Santa Maria), Santa Maria, v.39, n.1, p. 33-42, Jan./Jul.2013. Rodrigues, S.; et al. 
Conforme exposto, a enfermagem pode buscar junto à rede social de apoio, 0 engajamento fundamental para o cuidado à pessoa com estomia nas mais diversas situações, dentre elas 0 desenvolvimento e implementação de intervenções que trabalhe os sentimentos, emoções e atitudes em relação a si e aos outros.

Dessa forma, encaminhar e estimular a pessoa com estomia a participar de um grupo de apoio, que possa ajudar a conviver com esta nova situação é uma ação da enfermagem. Nos grupos são desenvolvidas possibilidade e habilidade do autocuidado, imprescindível para a reabilitação ${ }^{9}$. A satisfação na participação pode ajudar a superar a autorrejeição que influencia nos vários campos da vida, como o convívio social da pessoa como estomia, além de passar a vislumbrar um novo olhar em relação ao seu processo saúde-doença, produzindo novas interpretações e assegurando seu bem-estar por meio da identificação das mesmas dificuldades em outros seres humanos estomizados ${ }^{3}$.

0 profissional da saúde realiza todo o preparo no período anterior à confecção da estomia, orienta sobre o procedimento, sobre possíveis complicações e principalmente, fornece apoio emocional a pessoa e sua família. Além disso, o contato posterior ao procedimento cirúrgico poderá proporcionar adesão ao tratamento e melhor aceitação as novas condições de vida ${ }^{8}$. Assim, destaca-se o preparo do enfermeiro para o reconhecimento das redes de apoio, bem como se identificar como parte integrante desta.

\section{Considerações Finais}

Percebe-se a importância do enfermeiro conhecer os componentes da rede de apoio das pessoas com estomia e qual sua repercussão na vida dessa pessoa que está sendo cuidada. A partir disso, o fazer da enfermagem está no sentido de identificar as necessidades dos indivíduos e então orientar de forma clara e pertinente, e que juntos possam ser estabelecidas as estratégias para a melhoria da qualidade de vida dos envolvidos. Inserem-se nesse contexto os familiares e a sua importância como (co)responsáveis no cuidado, uma vez que a assistência de enfermagem deve englobar o trabalhar com e para a família.

Assim, observa-se a necessidade do cuidado de enfermagem voltado não somente à pessoa com estomia, mas sim ao seu contexto familiar, pautada no envolvimento entre a pessoa com estomia, o profissional, e o familiar. Essa assistência deve ser embasada no intuito de realizar a troca de experiências e saberes; planejar ações terapêuticas em conjunto; reconhecer as particularidades dos envolvidos; possibilitando, desse modo, uma melhora na qualidade de vida das pessoas e de seus familiares.

Os grupos ou associação de pessoas com estomia auxiliam no rompimento de paradigmas sociais existentes, fomentando a inclusão social e o desenvolvimento de melhores estratégias de enfrentamento do processo saúde-doença.

Os serviços e os profissionais de saúde precisam estar preparados, por meio do planejamento da assistência que inclua o apoio psicológico e a educação em saúde,

Saúde (Santa Maria), v.39, n.1, p. 33-42,

As redes sociais de apoio no cuidado às pessoas com estomias: Revisão Bibliográfica desenvolver as aptidões da pessoa para 0 autocuidado, contribuindo assim para a melhoria significativa da qualidade de vida destas pessoas.

Nesse sentido, uma série de aspectos importantes deve fazer parte do planejamento da assistência de enfermagem. Almeja-se que o enfermeiro preste um cuidado integral à pessoa com estomia, a fim de estabelecer vínculo e identificar particularidades, para formular um plano de cuidados que proporcione a reabilitação e reinserção dessa na sociedade. 
Diante disso, acredita-se que para os profissionais de saúde a identificação e compreensão das redes sociais de apoio no cuidado à pessoa com estomia são fundamentais para uma assistência de qualidade. Ainda, há necessidade de pesquisas e publicações acerca das demais redes de apoio, como a influência da espiritualidade no enfrentamento das dificuldades vivenciadas por eles. Este artigo, portanto, poderá contribuir como um recurso para a formação profissional e a educação continuada de enfermeiros, no sentido de compreender a singularidade e o bem-estar às pessoas com estomias, junto à rede social.

\section{Referências Bibliográficas}

1. Nascimento CMS, Trindade GLB, Luz MHBA, Santiago RF. Vivência do paciente estomizado: uma contribuição para assistência de enfermagem. Texto Contexto Enferm, Florianópolis, 2011 Jul-Set; 20(3): 55764.

2. Martins ML, Silva DGV, Silva RDM, Pereira VC, Crestani MM, D'avila FS. Pessoas Ostomizadas: A construção de seu itinerário terapêutico. Nursing (São Paulo). 2006; 8(99):953-957.

3. Barros EJL, Santos SSC, Erdmann AL. Rede social de apoio às pessoas idosas estomizadas à luz da complexidade. Acta paul enferm. 2008;21(4):595-601.

4. Brum, C.N, Sodré, BS, Prevedello, P. V, Quinhones, S.W.M. O processo de viver dos pacientes adultos com ostomias permanentes: uma revisão de literatura. Cuid. fundam. online 2010. out/dez. 2(4):1253-1263

5. Sluzki, C.E. A rede social na prática sistêmica. 2nd ed. São Paulo: Casa do Psicólogo; 2003

6. Cascais A FMV, Martini JG, Almeida PJ S. O impacto da ostomia no processo de viver humano. Texto contexto - enferm. [periódico na Internet]. 2007 Mar [citado 2012 Jan 11] ; 16(1): 163-167. Disponível em: http://www.scielo.br/scielo.php?script=sci_arttext\&pid=S0104-07072007000100021\&lng=pt.

http://dx.doi.org/10.1590/S0104-07072007000100021.

7. Maruyama,S.A.T ; Zago, M. M.F. O processo de adoecer do portador de colostomia por câncer. Revista. Latino-Americana de Enfermagem v.13 n.2 Ribeirão Preto Mar./Apr. 2005.

8. Santana JCB, Dutra BS, Tameirão MA, Silva PF, Moura IC, Campos ACV. O significado de ser colostomizado e participar de um programa de atendimento ao ostomizado. Cogitare enferm[periódico na

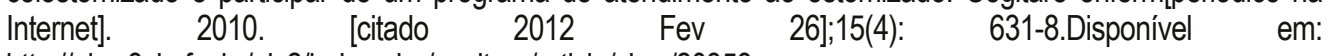
http://ojs.c3sl.ufpr.br/ojs2/index.php/cogitare/article/view/20358.

9. Souza SS,Vieira FA, Kerkoski E, Silva DMGV, Meirelles BHS, Baptista R et al. Redes sociais de pessoas com problemas respiratórios crônicos em um município do sul do Brasil. Cogitare enferm[periódico na

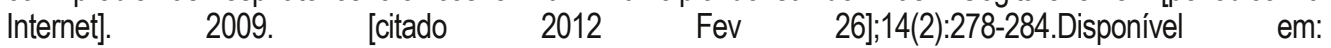
http://ojs.c3sl.ufpr.br/ojs2/index.php/cogitare/article/view/15619.

10. Minayo, M. C. S. O Desafio do Conhecimento. Pesquisa Qualitativa em Saúde. 12 ed. São Paulo. Hucitec, 2010.

11. Altschuler A, Ramirez M, Grant M, Wendel C, Hornbrook MC,Herrinton L,Krouse RS. The influence of husbands' or male partners' support on women's psychosocial adjustment to having an ostomy resulting from colorectal cancer. J Wound Ostomy Continence Nurs [periódico na Internet]. 2009. [citado 2012 Jan 11];36(3):299-305. Disponivel em:

http://www.ncbi.nlm.nih.gov/pmc/articles/PMC2806676/?tool=pubmed

12. Sampaio FAA, Aquino PS, Araújo TL, Galvão MTG. Assistência de enfermagem a paciente com colostomia: aplicação da teoria de Orem. Acta paul. enferm. [periódico na Internet]. $2008 \mathrm{Mar}$ [citado 2012 Jan 11] ; 21(1): 94-100. Disponível em: http://www.scielo.br/scielo.php?script=sci_arttext\&pid=S010321002008000100015\&lng=pt. http://dx.doi.org/10.1590/S0103-21002008000100015.

Rev. Saúde (Santa Maria), Santa Maria, v.39, n.1, p. 33-42, Jan./Jul.2013. Rodrigues, S.; et al. 
13. Silva AL, Shimizu HE. A relevância da Rede de Apoio ao estomizado. Rev. bras. enferm. [periódico na Internet]. 2007 Jun [citado 2012 Jan 11] ; 60(3): 307-311. Disponível em: http://www.scielo.br/scielo.php?script=sci_arttext\&pid=S0034-71672007000300011\&lng=pt.

http://dx.doi.org/10.1590/S0034-71672007000300011.

14. Silva A L, Shimizu H E. O significado da mudança no modo de vida da pessoa com estomia intestinal definitiva. Rev. Latino-Am. Enfermagem [periódico na Internet]. 2006 Ago [citado 2012 Jan 11] ; 14(4): 483490. Disponível em: http://www.scielo.br/scielo.php?script=sci_arttext\&pid=S010411692006000400003\&lng=pt. http://dx.doi.org/10.1590/S0104-11692006000400003.

15. Slater RC. Managing quality of life in the older person with a stoma. $\mathrm{Br} \mathrm{J}$ Community Nurs[periódico na Internet]. 2010. [citado 2012 Jan 11]; 15(10):480- 484. Disponível em: http://www.internurse.com/cgibin/go.pl/library/article.cgi?uid=78728;article=BJCN_15_10_480_484

16. Martins ML, Silva RDM, Fangier A, Perugini VC, Pereira VC, D'Ávila FS et al . A trajetória do grupo de apoio à pessoa ostomizada: projetando ações em saúde e compartilhando vivências e saberes. Texto contexto - enferm. 2005 Dez; 14(4): 594-600.

17. Mowdy S. The role of the WOC nurse in an ostomy support group. J Wound Ostomy Continence Nurs[periódico na Internet].1998. [citado 2012 Jan 11];25(1):51-4. Disponível em:http://ac.elscdn.com/S1071575498900134/1-s2.0-S1071575498900134-

main.pdf?_tid=f2cf2c9f4471af9c1baf5908e007be3e\&acdnat=1336742705_95bca498f3776fa8385342bba8f6 $5 a 7 d$

18. Trentini M, Silva D, Pacheco M, Martins M. Parceria - uma estratégia para promoção da saúde. Cogitare Enfermagem[periódico na Internet]. 1996. [citado 2012 Jan 11]; 1(2): 8-10. Disponível em: http://ojs.c3sl.ufpr.br/ojs2/index.php/cogitare/article/view/8728/6050.

19. Borwell B. Continuity of care for the stoma patient: psychological considerations. $\mathrm{Br} \mathrm{J}$ Community Nurs[periódico na Internet]. 2009. [citado 2012 Jan 11];14(8):326-331. Disponível em: http://www.internurse.com/cgi-bin/go.pl/library/article.cgi?uid=43511;article=BJCN_14_8_326_331

20. Williams J. Stoma care nursing: what the community nurse needs to know. $\mathrm{Br} \mathrm{J}$ Community Nurs [periódico na Internet]. 2007. [citado 2012 Jan 11];12(8):342-6. Disponível em: http://www.internurse.com/cgibin/go.pl/library/article.cgi?uid=24361;article=BJCN_12_8_342_346

21. Souza, J.L de, Gomes, G.C, Barros, E.J.L. O cuidado à pessoa portadora de estomia: o papel do familiar cuidador. Rev. enferm. UERJ, Rio de Janeiro, 2009 out/dez; 17(4):550-5.

\section{Sandra Ost Rodrigues Martins Carvalho}

Endereço para correspondência - Rua Bernardo Zamperete, 30, Apto 401. Bairro Maria Alice Gomes, Santiago, RS, Brasil.

E-mail: sandrinhaost@yahoo.com.br

Currículo Lattes: http://lattes.cnpq.br/1042104084556576

Recebido em 21 de novembro de 2012

Aprovado em 23 de julho de 2013

Saúde (Santa Maria), v.39, n.1, p. 33-42,

2013.

com estomias: Revisão Bibliográfica 